\title{
The Current Phenomenon in the Indium-Tin Oxide-Alignment Layer-Liquid Crystal Structure
}

\author{
Z. Mykytyuk*, A. Fechan, O. Sushynsky, O. Yasynovska and V. Kotsun \\ Lviv Polytechnic National University, 79013 Lviv, Ukraine
}

\begin{abstract}
This work is connected to investigation of frequency dependences of conductive value and dielectric conductivity of the indium-tin oxide-alignment layer-liquid crystal structure which allows to find new ways in formation of the electrically stabilized structure of liquid crystal materials for the optoelectronic devices.
\end{abstract}

PACS numbers: $61.30 . \mathrm{Gd}, 42.82 . \mathrm{Gw}, 83.80 . \mathrm{Xz}$

\section{Introduction}

At the present day the indium-tin oxide-alignment layer-liquid crystal structures find wide applications in informational display devices, in adaptive optics and in control devices of optical signal. Field electrooptical effects are commonly used in such devices but for effective operation the conductivity and dielectric permeability of the liquid crystal play a huge role. Especially, this problem is actual for liquid crystal materials with negative anisotropy of dielectric conductivity when it is necessary to form complex textures stabilized by external electrical field.

\section{Results and discussion}

For realization of above-mentioned method we propose to use sandwich type cell with initial homeotropic alignment, which is filled by nematic liquid crystal with negative dielectric anisotropy $(\Delta \varepsilon<0)$ and the positive anisotropy of refractive index $(\Delta n>0)$. The cross-section of this structure is shown in Fig. 1.

With no electrical field applied, the liquid crystal layer is homogeneous in the direction of light propagation and has refractive index which is equal to $n_{\perp}$. When electrical field is applied to the structure the liquid crystal molecules are re-orientated and formation of planar oriented region at the center is observed. The refractive index in the direction of light propagation is $n_{\|}>n_{\perp}$. Thus, liquid crystal layer under applied electrical field possesses waveguide properties, homeotropically aligned near-surface regions operate as cladding, and planar oriented region close to the center operates as the core. The main advantage of this solution is complete formation of planar waveguide inside the liquid crystal layer [1]. It allows to widen the range of materials that are used to form plates and the liquid crystal is placed between

\footnotetext{
* corresponding author; e-mail: zmykytyuk@polynet.lviv.ua
}

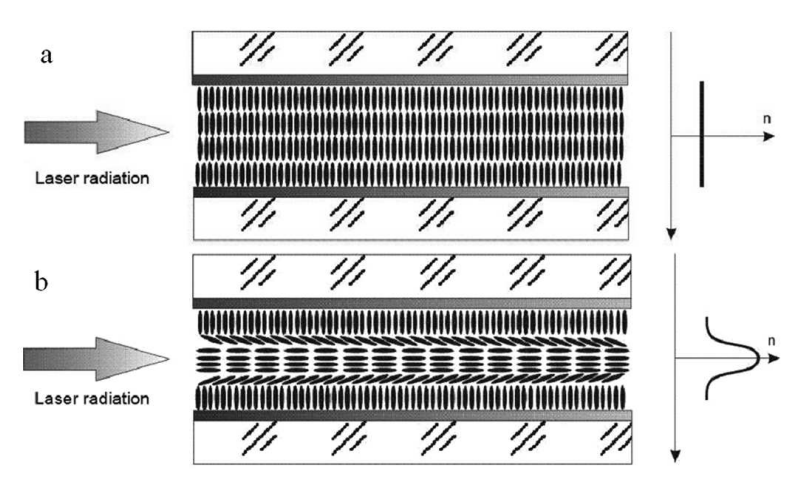

Fig. 1. Cross-section of the sandwich type cell and a distribution of the refractive index: (a) with initial homeotropic alignment of nematic liquid crystal; (b) deformation of nematic texture under electrical field.

these plates. These plates do not involve in the process of light propagation through this structure and their optical properties do not play an important role. Another advantage of this structure is the ability to control planar waveguide properties by electrical field. We can also control waveguide presence or absence inside the liquid crystal layer.

We have chosen the nematic liquid crystal $N$-(4methoxybenzylidene)-4-butylaniline (MBBA) and transparent conductive layer of indium-tin oxide (ITO) as investigation objects. For the formation of initial alignment of liquid crystal molecules we have used the polymer layer PI 2555 (plane alignment) and T9039 PI 2555 (homeotropic alignment) and lecithin. The main feature of the chosen liquid crystal material is the negative dielectric anisotropy and the positive anisotropy of refraction index. The investigations were carried out with sandwich type cell and the liquid crystal layer thickness of $20 \mu \mathrm{m}$.

One of the most effective method for registration of the direct field deformation in nematic liquid crystal layer is the conoscopy. The main point of this method is the observation of fringe pattern of a beam which passed via 
liquid crystal cell that is placed between two crossed polarizers [2]. The block-diagram of conoscopic experimentation is shown in Fig. 2.

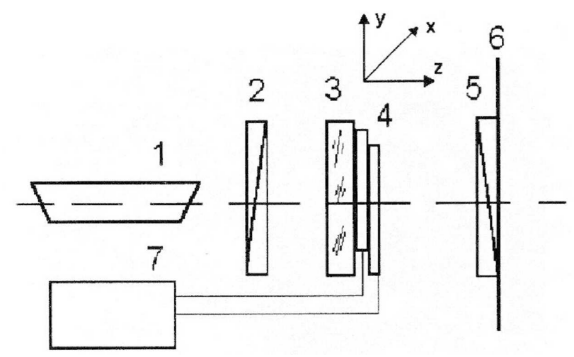

Fig. 2. The block diagram for registration of the conoscopic pattern: 1 - laser, 2 - polarizer, 3 - scattering plate, 4 - liquid crystal cell, 5 - analyzer, 6 - screen, 7 - power supply unit.

The main problem which appears during texture formation is the hydrodynamic instability caused by transient (nonstationary) ion drift in the liquid crystal layer $[3,4]$.

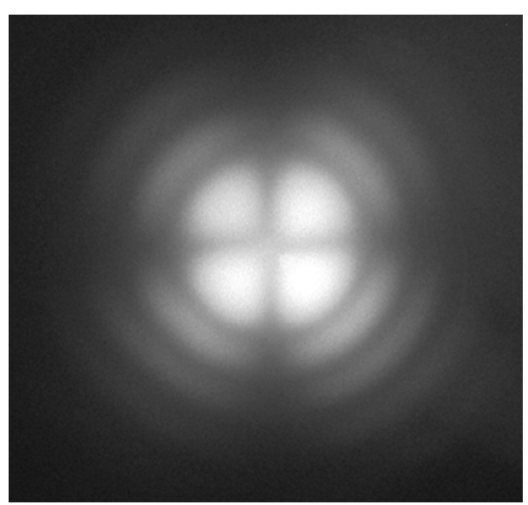

Fig. 3. The experimental conoscopic pattern of initial homeotropic-aligned sample at $U=0 \mathrm{~V}$.

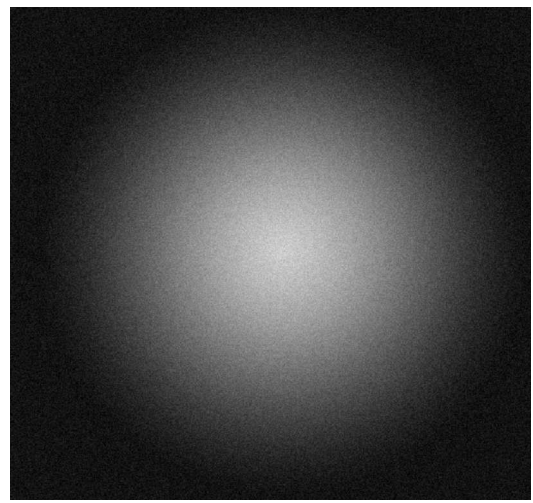

Fig. 4. The experimental conoscopic pattern at electrohydrodynamic instability of the sample $U=4 \mathrm{~V}$.
The experimental conoscopic patterns obtained for initial homeotropic alignment of liquid crystal molecules are shown in Figs. 3 and 4 . The homeotropic alignment is obtained by lecithin introduction into nematic material at different control voltages and the frequency of $1 \mathrm{kHz}$.

The experimental conoscopic pattern which corresponds to initial homeotropic alignment of the liquid crystal layer is shown in Fig. 3. Such conclusion can be made from the fringe pattern that has a typical dark cross formed by isogyres with isochromatic rings around, and complete fringe pattern which possesses symmetry of rotation around optic axis. The rotation of the sample in the plane, that is perpendicular to the direction of light propagation, does not lead to change in conoscopic pattern and it indicates absence of initial inclination of the optic axis of the nematic sample. The applying of the control voltage to the sample leads to conoscopic pattern vanishing, formation of homogeneous pattern with reduction of radiation intensity from center to the boundary, and it indicates absence of long axis order in the nematic liquid crystal sample (Fig. 4). Such behaviour of the sample can be explained by initiation of electrohydrodynamic instability (EHDI) phenomena caused by transient (nonstationary) ion drift in the sample.

We used dielectric polymer layers deposited on ITO surface to eliminate EHDI phenomena. These polymer layers also help along assigning of the initial homeotropic alignment.

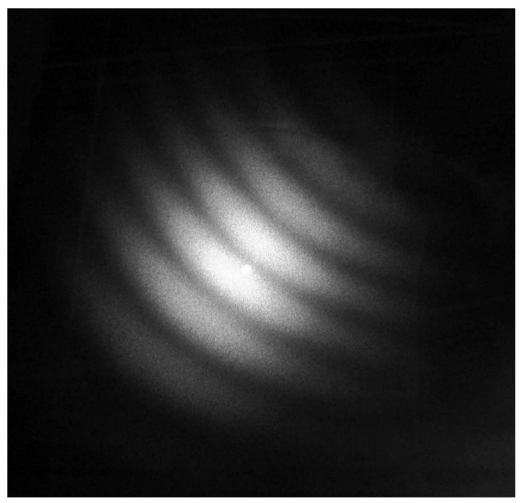

Fig. 5. The experimental conoscopic pattern of the sample with planar-oriented central region at $U=4 \mathrm{~V}$.

Experimental conoscopic patterns obtained for the initial homeotropic alignment of liquid crystal molecules are shown in Figs. 5 and 6. The initial homeotropic alignment was formed by dielectric oriented layer at different values of control voltage and the frequency of $1 \mathrm{kHz}$. The initial homeotropic alignment pattern for these samples was identical to the pattern in Fig. 3. However, the applying of the control voltage, in this case, causes the obtaining of series of conoscopic patterns at the control voltage range of $0-20 \mathrm{~V}$.

Realignment of long axis of molecules in the central region of the liquid crystal layer (for a small value of a control voltage) leads to change in fringe pattern and 


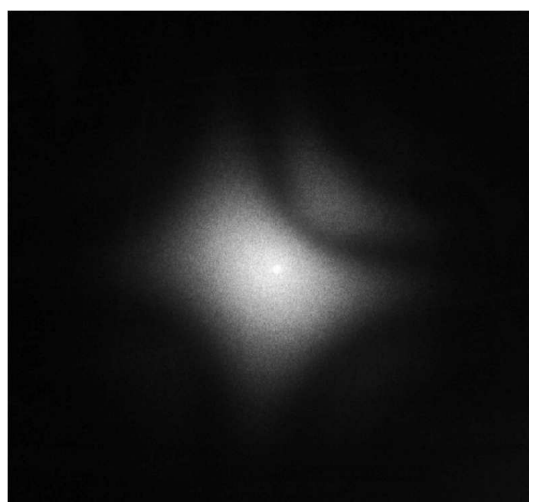

Fig. 6. The experimental conoscopic pattern of planar oriented sample at $U=20 \mathrm{~V}$.

the cross displacement from the center of visual field in the direction of realignment of long axis of nematic molecules is observed. Further increase of applied voltage (in the range of $2-15 \mathrm{~V}$ ) causes a broadening of the planar-aligned zone in the liquid crystal (Fig. 4). The further voltage increase causes the appearing of isochromatic fringes of hyperbolic type which are typical for nematic planar alignment (Fig. 5). But the absence of pattern symmetry relatively to light propagation axis $(U=20 \mathrm{~V})$ indicates a presence of thin near-surface homeotropic-aligned layers inside the sample. Further voltage increase does not change the conoscopic pattern.

We also carried out the investigations of conductivity and dielectric anisotropy. They were carried out in a sandwich type cell with the liquid crystal layer thickness of $20 \mu \mathrm{m}$ using the impedance spectroscopy in the frequency range of $10^{3}-10^{5} \mathrm{~Hz}$, and using the measuring complex "mAUTOLAB" made by "ECO CHEMIE" (Holland), and FRA-2 and GPES softwares.

The dependence of resistivity active component for different method of initial alignment formation is shown in Fig. 7. The resistivity value does not depend on ini-

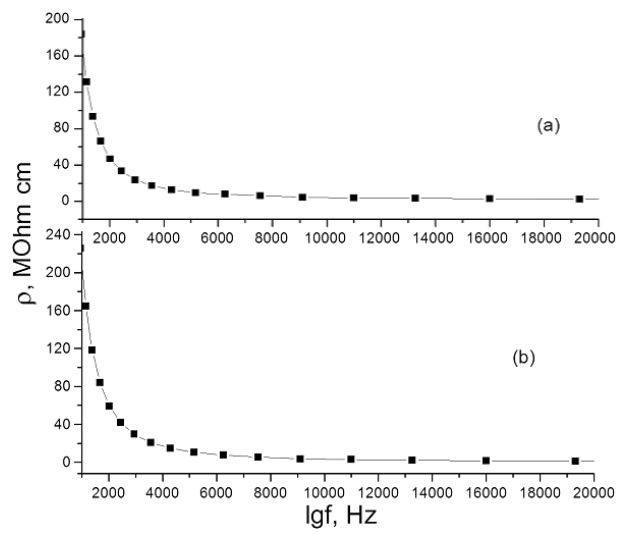

Fig. 7. Frequency dependences of the resistivity of the active component of liquid crystal cells with lecithin (a) and dielectric aligned (b) layers.

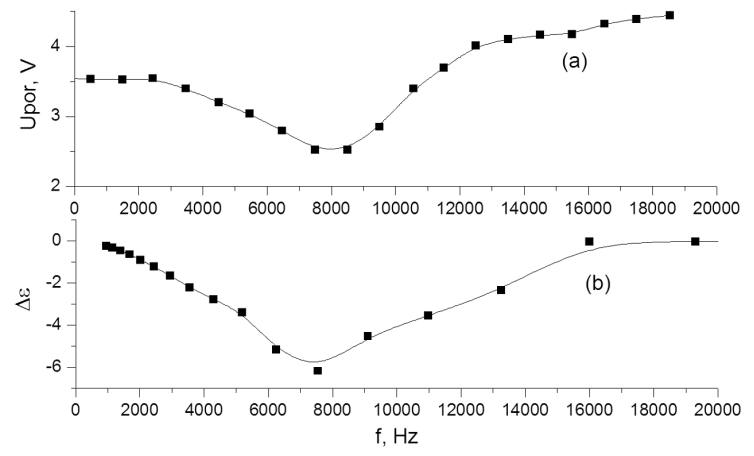

Fig. 8. Frequency dependences of the voltage when homeotropic texture of the sample is vanished (a), and of dielectric anisotropy active component (b).

tial alignment formation method. It allows to state that ion drift mechanism is not important in the investigated frequency range.

The investigations of frequency dependences of dielectric anisotropy active component (Fig. 8) show that minimum is observed at the voltage frequency of $7.5 \mathrm{kHz}$ in the investigated range. Received results are in good agreement with a vanishing voltage for nematic homeotropic texture. The vanishing voltage minimum is also observed in the range of $7.7-8.5 \mathrm{kHz}$.

\section{Conclusions}

The application of dielectric polymer oriented layers in comparison with lecithin allows the formation of more perfect hybrid texture at identical control pulse parameters. It can be explained by the reduction of the influence of transient ion drift on perfection of the liquid crystal layer texture when dielectric polymer alignment layers are used.

The change of the control pulse frequency allows to change the control voltage in the range of $2.5-4.5 \mathrm{~V}$. It is shown that a main influence on vanishing voltage for initial homeotropic structure has the frequency dependence of dielectric permittivity anisotropy.

\section{References}

[1] Pat. No. u 2008625 Ukraine, G02F 1/13. The method of making of integral microelectronic solid-state device/Hotra Z. [et al.]: assigned to Lviv Polytechnic National University, date of patent: December 29 (2008).

[2] Ya. Nastishin, O. Dovgyj, O. Vlokh, Ukr. J. Phys. Opt. 3, 98 (2002).

[3] I. Guralnik, V. Belopuhov, G. Love, A. Naumov, J. Appl. Phys. 87, 4069 (2000).

[4] H. Naito, Y. Yokoyama, S. Murakami, M. Imai, M. Okuda, A. Sugimura, Mol. Cryst. Liq. Cryst. 262, 249 (1995). 